\title{
BMJ Open Effectiveness of community-based health services by nurse practitioners: protocol for a systematic review and meta-analysis
}

\author{
Mikiko Kanda, ${ }^{1}$ Erika Ota, ${ }^{2}$ Hiromi Fukuda, ${ }^{3}$ Shinji Miyauchi, ${ }^{3}$ Stuart Gilmour, ${ }^{1}$ \\ Yuko Kono, ${ }^{3}$ Erika Nakagama, ${ }^{3}$ Sachiyo Murashima, ${ }^{3,4}$ Kenji Shibuya ${ }^{1}$
}

To cite: Kanda M, Ota $\mathrm{E}$, Fukuda $\mathrm{H}$, et al. Effectiveness of community-based health services by nurse practitioners: protocol for a systematic review and metaanalysis. BMJ Open 2015;5: e006670. doi:10.1136/ bmjopen-2014-006670

- Prepublication history and additional material is available. To view please visit the journal (http://dx.doi.org/ 10.1136/bmjopen-2014006670)

Received 25 September 2014 Revised 12 March 2015 Accepted 14 April 2015

CrossMark

For numbered affiliations see end of article.

Correspondence to

Mikiko Kanda;

mkanda@m.u-tokyo.ac.jp

\section{ABSTRACT}

Introduction: To realise universal health coverage in an ageing society, adequate provision of appropriately trained human resources is essential. The nurse practitioner (NP) is an autonomous and independent, advanced practice nurse capable of providing treatment and care that can be substituted for some aspects of a medical doctor's (MD's) role, especially in a community setting. Previous systematic reviews found higher levels of patient satisfaction with services provided by NPs than those provided by MDs. As noncommunicable diseases become a major health burden requiring long-term healthcare in community settings, this systematic review aims to assess the equivalence of NP services to standard care provided by MDs, and to determine whether their practice is an effective alternative to that of MDs in community settings

Methods and analysis: Relevant randomised controlled trials (RCTs) and cluster RCTs will be searched in the Cochrane Central Register of Controlled Trials, MEDLINE, EMBASE, CINAHL and the British Nursing Index. We will assess patient and health system utilisation outcomes of interventions comparing treatment and care provided by NPs in community settings with that provided by MDs. Two authors will independently screen studies for inclusion, consulting with a third author where necessary to resolve discrepancies. The risk of bias of included studies will be assessed using the Cochrane Collaboration risk of bias tool, and quality of evidence using the GRADE approach. Meta-analysis of included studies will be conducted using fixed-effect or random-effects models depending on the degree of between-study heterogeneity. Results will be presented using risk ratios with $95 \% \mathrm{Cl}$ for dichotomous outcomes and standardised mean differences with $95 \% \mathrm{Cl}$ for continuous outcomes.

Ethics and dissemination: This systematic review and meta-analysis protocol does not require ethical approval. We will disseminate the findings of this systematic review and meta-analysis via publications in peer-reviewed journals.

Trial registration number: PROSPEROCRD420 14009627.

\section{INTRODUCTION}

The percentage of the world's population aged 60 years and above is estimated to double from approximately $11 \%$ to $20 \%$ by $2050 .{ }^{1}$ To realise and sustain universal health coverage (UHC) in an ageing society, adequate provision of well-prepared human resources for health (HRH) is essential. ${ }^{2}$ Nurses constitute the largest profession in the world, ${ }^{3}$ and are the frontline-often the only -healthcare personnel available to the population, especially in a community setting. It is therefore important to secure a practical environment that enables nurses to optimise their expertise to provide a high quality of services.

A nurse practitioner (NP) is a type of advanced practice nurse (APN) defined by the International Council of Nurses as "a registered nurse who has acquired an expert knowledge base, complex decisionmaking skills and clinical competencies. A master's degree is recommended for entrylevel positions". ${ }^{4}$ Although many countries have introduced a NP system, the status of education, regulations, code of practice and competencies, vary substantially across countries and regions. ${ }^{5}$ Many countries with limited HRH are seeking ways to improve the efficiency of healthcare delivery, and utilising NPs is one solution that may enable the provision of primary healthcare with advanced scope of practice. For instance, in a community setting where NPs are the first point of contact, such as at a nursing home, geriatric healthcare facility, home-visit nursing agency, in the home or at the clinic, the NP performs assessments and diagnoses, orders diagnostic and laboratory tests, prescribes medication and offers treatments with a high level of autonomy and independence. Also taking responsibility for case management, the NP 
monitors patient health and medical plan adherence, offers counselling and education for non-communicable disease (NCD) prevention, ensures continuity of care and manages hospital readmission. The NP is also responsible for disease symptom management and is expected to show advanced consultation, collaboration, education, research and leadership skills. ${ }^{4}$

With NCDs becoming a major burden on population health globally, ${ }^{7}$ the credentials and competencies of NPs may be beneficial in the management of NCDs, which requires long-term care in primary-care settings, especially in countries with an increasing ageing population. Moreover, NPs are in charge of managing individual health in communities with few medical doctors (MD) ${ }^{8}$ It is essential to assure that NP practice is sufficiently effective to make up for the shortage of MDs, and/or can be equivalent to care provided by MDs in a community setting.

Two comprehensive systematic reviews have previously assessed NP practice. ${ }^{9}{ }^{10}$ One review, conducted in 2002, examined the equivalence of services provided by NPs and by MDs in primary care. ${ }^{9}$ This systematic review and meta-analysis of 11 trials and 23 observational studies identified higher levels of patient satisfaction with services provided by NPs than those provided by MDs, and no significant difference in patient health status, prescriptions and return consultations. The other review quantified APN outcomes, including NPs, from articles published in the USA between 1990 and 2008. ${ }^{10}$ This study identified 14 trials including 12 high quality scaled studies and 23 observational studies. From these trials, NP practice outcomes were summarised in dimensions of patient satisfaction, self-rated health, physical function and biological data such as blood sugar control, lipid control and blood pressure. These outcomes were compared with the same outcomes in patients whose care was managed exclusively by MDs. However, no systematic review or meta-analysis has focused on NP practice specifically in a community setting. As services provided by NPs vary depending on the setting, we will conduct a systematic review and meta-analysis focusing on NP practice in the community settings.

\section{Objectives}

The main objective of this study is to investigate whether services delivered through substitution of NPs for MDs result in statistically equivalent patient and health system utilisation outcomes compared to standard care provided by MDs in a community setting.

\section{METHODS}

\section{Type of studies}

Randomised controlled trials (RCTs) and cluster RCTs of interventions comparing NPs and MDs will be included. We will not include quasi-RCTs and cross-over trials.

This review protocol has been registered with the International Prospective Register of Systematic Reviews
(PROSPERO) at the National Institute for Health Research and Center for Reviews and Dissemination (CRD) at the University of York (registration number: CRD42014009627).

\section{Type of participants}

The participants will be adults receiving treatment and care from NPs in a community setting. Community settings include nursing homes, geriatric healthcare facilities, home-visit nursing agencies, patient homes and clinics that cover all areas except hospital inpatients.

\section{Type of intervention}

The types of interventions by NPs will be as follows:

- As a first point of contact for patients or clients, performing assessments, and ordering diagnostic and laboratory tests;

- Offering diagnoses, and prescribing medications and treatments;

- Implementing procedures;

- Taking responsibility for case management;

- Following up and monitoring patient health and medical plan adherence;

- Counselling and providing education for preventing NCDs;

- Ensuring continuity of care and hospital re-admission;

- Disease symptom management.

All interventions are provided by NPs in a community setting.

\section{TYPE OF OUTCOME MEASURES \\ Primary outcomes}

1. Hospitalisation (times/year)

2. Patient mortality

3. Biological data: cholesterol level (g), blood pressure $(\mathrm{mm} \mathrm{Hg})$, blood sugar $(\mathrm{mg} / \mathrm{dL})$ and glycated haemoglobin (\%)

\section{Secondary outcomes}

1. Cost (International dollars or US dollars)

2. Patient satisfaction

3. Self-reported perceived health

4. Pressure ulcers

5. Functional status (Activities of Daily Living (ADL)/ Instrumental Activities of Daily Living (IADL))

6. Emergency department visits (times/year)

7. Length of hospital stay (days)

\section{Search strategy and sources}

We will report data following the preferred reporting items for systematic review and meta-analysis (PRISMA) statement. ${ }^{11} \mathrm{~A}$ comprehensive literature review using the databases MEDLINE, EMBASE, CINAHL, British Nursing Index and Cochrane Central Register of Controlled Trials (CENTRAL) will be performed; an example of search strategy in MEDLINE is shown in online supplementary file 1 . Search strategies will be 
tailored to each database so as to employ the correct Medical Subject Headings (MeSH) terms. Where possible, MeSH and free text terms with synonyms will both be used so as to increase identification of potentially relevant studies. Where MeSH terms are not used, free text only will be used. A separate search of Web of Science will be undertaken in order to capture any grey literature. Reference list reviews of included papers will be carried out. No language restrictions will be applied to the searches.

\section{DATA COLLECTION AND ANALYSIS Inclusion criteria}

1. Participants: Adult patients who received treatment and care by NPs or standard care by MDs in the community setting.

2. Study design: RCTs including cluster RCTs

3. Intervention site: Community setting including nursing homes, geriatric medical care facilities, geriatric healthcare facilities, home-visit nursing agencies, patient homes and clinics.

4. Intervention: All types of treatment and care provided by NPs in a community settings.

5. High-income countries based on World Bank criteria in 2013.

6. Countries that require NPs to hold a master's degree at the time of the study period. If education qualifications are not clearly mentioned, detailed information will be obtained by contacting authors of the article or by reference to established qualification standards for the country in question where the study clearly specifies that NPs are defined with reference to national accreditation boards.

7. Published original articles (full-text available including theses) published from 1990 to 2014. The time period was chosen because the scientific and organisational basis of clinical practice and intervention changed in1990. ${ }^{10}$

\section{Exclusion criteria}

1. Excluded studies: Observational studies, quasi-randomised and cross-over trials.

2. Excluded intervention sites: Inpatient care at hospitals.

3. Excluded participants: Children.

4. Excluded publications: Non-academic articles and articles published before 1990.

\section{Data extraction and management}

The study title and abstract will be screened by two authors in the review group independently to identify eligible studies. Two authors in the study group will manually enter data into a standard data extraction form based on the Cochrane Handbook for Systematic Reviews of Interventions, ${ }^{12}$ to determine the eligibility of each study. Any disagreement will be resolved by discussion. If there is any discrepancy between the two authors, we will consult with other authors (EO, SaM and KS) for expert opinion. When there is unclear information in the process of data extraction, we will contact the authors of the original studies to provide further information.

\section{Assessment of risk of bias in included studies}

The risk of bias in included studies will be assessed using the Risk of Bias tool according to the Handbook. ${ }^{12}$ We will use the following criteria to assess the risk of bias: random sequence generation, allocation sequence concealment, blinding of participants and personnel, blinding of outcome assessment, incomplete outcome data, selective outcome reporting and other bias. ${ }^{12}$ Studies will be included in meta-analysis if they are of the same type, such as RCTs or cluster RCTs, and have the same population, intervention, comparison and outcomes. Evaluation of whether or not included studies are eligible for meta-analysis will be conducted by four authors (MK, EO, HF and SG), and in the event of disagreement, we will consult with other authors (EO, SaM and KS) for expert opinion.

\section{Measurement of treatment effect}

Statistical analysis will be carried out using RevMan $2014{ }^{13}$ For dichotomous outcomes including hospitalisation, patient mortality and emergency department use, risk ratios with $95 \%$ CIs will be used to assess differences in the outcomes of treatment and care provided by NPs compared to MDs. For continuous outcomes including biological data, cost, patient satisfaction, self-reported perceived health and functional status (ADL/IADL), standardised mean differences with $95 \%$ CI will be calculated.

\section{Missing data}

We will assess levels of attrition for included studies, and conduct sensitivity analysis of the impact of including studies with $20 \%$ or more of missing data. For all outcomes, we will conduct intention-to-treat analysis wherever possible.

\section{Assessment of publication bias}

If a sufficient number (10 or more) of studies are eligible for meta-analysis, funnel plots will be used in order to assess reporting bias by checking funnel plot asymmetry.

\section{Strategy for data synthesis, assessment/investigation of heterogeneity}

We will use a fixed-effect model for combining data if the interventions examined in the studies are judged to be the same based on the heterogeneity between studies, and if the methods are fairly similar. We will use a random-effects model when the interventions in the studies are considered to have clinical heterogeneity or there is substantial heterogeneity between studies. The results of the random-effects model will be used as the average range of possible intervention effects with $95 \%$ CIs, and the estimates of $\tau^{2}$ and $\mathrm{I}^{2}$ and difference of 
clinical implication between interventions will be discussed. Finally, we will assess the quality of the following individual outcomes and produce summaries using the GRADE approach:

1. Hospitalisation

2. Patient mortality

3. Biological data

4. Cost

5. Patient satisfaction

6. Self-reported perceived health

Data will be imported from RevMan $2014^{13}$ to the GRADE profiler ${ }^{14}$ to produce 'summary of findings' tables. These tables will include a summary of the intervention effect and a quality of individual outcomes using the GRADE approach. The quality of the body of evidence for each outcome will be assessed based on five factors: study limitations, consistency of effect, imprecision, indirectness and publication bias.

\section{Analysis of subgroups or subsets}

If any substantial heterogeneity is identified through analysis of $\tau^{2}$ and $\mathrm{I}^{2}$ statistics, subgroup analysis will be conducted for primary outcomes in the following characteristic groups

1. Type of facility: Geriatric healthcare facilities, homevisit nursing agencies, clinics and hospitals

2. Gender: Males versus females

3. Age group: Less than 40 years vs 40 years and over

4. Type of intervention

5. The number of NPs delivering the intervention: less than 10 vs 10 and over

6. The years of NP experience: less than 10 years and 10 years and over

Subgroup differences will be assessed by interaction tests. The results of subgroup analyses will be reported quoting the $\mathrm{I}^{2}$ statistic and $\mathrm{p}$ value, and the interaction test $\mathrm{I}^{2}$ value.

\section{DISCUSSION}

This review and meta-analysis will play an important role in consolidating evidence on the effectiveness of health services provided by NPs, especially where they play a role in managing NCD, supporting continuity of care between hospital and community, and monitoring and supporting the health of elderly people. Information on which NP activities are effective as a substitute for standard care provided by MDs in terms of patient and health system utilisation outcomes will further drive efforts to develop effective NP utilisation strategies. These strategies in turn will strengthen support to and sustain UHC through provision of high quality care at low cost in community settings.

\section{Author affiliations}

${ }^{1}$ Department of Global Health Policy, Graduate School of Medicine,

The University of Tokyo, Tokyo, Japan
${ }^{2}$ Division of Clinical Practice Policy, Department of Health Policy, National Center for Child Health and Development, Tokyo, Japan

${ }^{3}$ Department of Nursing, Oita University of Nursing and Health Sciences, Oita, Japan

${ }^{4}$ The University of Tokyo, Tokyo, Japan

Acknowledgements The authors would like to thank Ms Miwako Segawa for devising a search strategy for this review.

Collaborators Dr Miho Tonai, Dr Miki Ono and Mr Yusuke Makino.

Contributors MK conceived and designed the review, completed the PROSPERO registration and wrote the primary draft. EO and HF conceived and designed the review, provided content expertise and revised the manuscript. SG provided content expertise in the statistical analysis section and revised the manuscript. ShM, YK and EN were involved in the design of the review, setting the outcomes of review, inclusion and exclusion criteria. SaM and KS contributed to content expertise and feedback, and provided important intellectual content. All authors read and approved the final version of the protocol for submission.

Funding This project is funded by the Japanese Society for Promotion of Science Grant in Aid for Scientific Research (B) 26293480 and National Center for Child Health and Development grant 26A-5.

Competing interests None.

Provenance and peer review Not commissioned; externally peer reviewed.

Open Access This is an Open Access article distributed in accordance with the Creative Commons Attribution Non Commercial (CC BY-NC 4.0) license, which permits others to distribute, remix, adapt, build upon this work noncommercially, and license their derivative works on different terms, provided the original work is properly cited and the use is non-commercial. See: http:// creativecommons.org/licenses/by-nc/4.0/

\section{REFERENCES}

1. World Health Organization. Global health and ageing. 2011. http:// www.who.int/ageing/publications/global health.pdf?ua $=1$

2. Sheikh M, Boerma T, Cometto $\mathrm{G}$, et al. Human resources for universal health coverage: a call for papers. Bull World Health Organ 2013;91:84-84A.

3. Hughes F. Nurses at the forefront of innovation. Int Nurs Rev 2006;53:94-101.

4. International Council of Nurses. Nurse practitioner/advanced practice nurse: definition and characteristics. Nurs Matters 2004; http://www. icn.ch/images/stories/documents/publications/fact_sheets/1b_ FS-NP APN.pdf

5. Delamaire M.-L, Lafortune G. Nurses in advanced roles. OECD Health Working Papers, OCDE, 2010;(54).

6. Sherwood GD, Brown M, Fay V, et al. Defining nurse practitioner scope of practice: expanding primary care services. Internet $J A d v$ Nurs Pract 1997;1:1-12.

7. Lozano R, Naghavi M, Foreman K, et al. Global and regional mortality from 235 causes of death for 20 age groups in 1990 and 2010: a systematic analysis for the Global Burden of Disease Study 2010. Lancet 2013;380:2095-128.

8. Naylor MD, Kurtzman ET. The role of nurse practitioners in reinventing primary care. Health Affairs 2010;29:893-9.

9. Horrocks S, Anderson E, Salisbury C. Systematic review of whether nurse practitioners working in primary care can provide equivalent care to doctors. BMJ 2002;324:819-23.

10. Newhouse RP, Stanik-Hutt J, White KM, et al. Advanced practice nurse outcomes 1990-2008: a systematic review. Nurs Econ 2011;29:230-50.

11. Moher D, Liberati A, Tetzlaff J, et al. Preferred reporting items for systematic reviews and meta-analyses: the PRISMA statement. Ann Intern Med 2009;151:264-9.

12. Higgins JP, Green S. Cochrane handbook for systematic reviews of interventions. Vol. 5. Wiley Online Library, 2008.

13. The Cochrane Collaboration. Review Manager (RevMan) [Computer program]. Version 5.3. 2014.

14. The Cochrane Collaboration. GRADEpro [Computer program] Version 3.6. 2008 